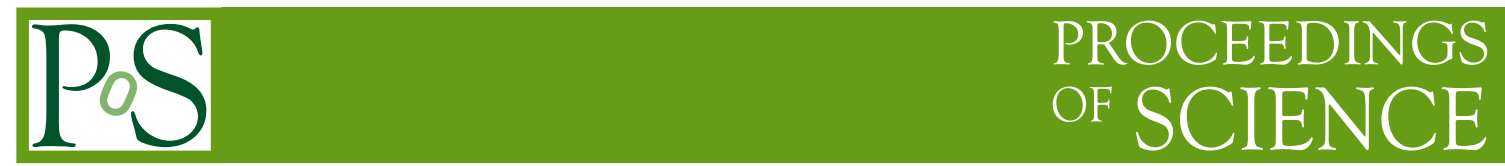

\title{
Effective Field Theories and Lattice for Hard Probes
}

\section{Nora Brambilla ${ }^{a, *}$}

${ }^{a}$ Physik Department, Technische Universität München, James-Franck-Strasse 1, 85748 Garching, Germany

E-mail: nora.brambilla@ph.tum.de

Effective Quantum Field Theories and QCD Lattice methods have become more and more complementary and mutually supportive in the study of Hard Probes. I present some of the progress that this alliance already delivered and I discuss future opportunities.

HardProbes 2020

1-6 June 2020

Austin, Texas

${ }^{*}$ Speaker 


\section{Introduction}

Effective Field Theories (EFTs) and QCD Lattice methods have become more and more complementary and mutually supportive in the study of Hard Probes. Since the physical problem is tremendously complex and should be addressed in the underlying field theory which is QCD, the combination of EFTs and lattice has allowed us to make big progress in the last times. On one hand, the EFT is pulling out scales from observables in a controlled way, separating them. On the other hand, the low energy contributions that the EFT has factorized can be evaluated on the lattice. This greatly reduces the complexity of the problem allowing the lattice to target the nonperturbative part directly, appropriately defined in the EFT in terms of gauge invariant, purely gluonic objects, a big simplification with respect to an ab initio lattice calculation of an observable. This last is much more difficult because still contains all the physical scales of the problem. In this framework also quenched lattice calculations can be pretty useful because at least at higher energy the flavor dependence is accounted for by the EFT matching coefficients. In relation to hard probes it has payed off to combine different EFTs, those who describe the hot medium properties like Hard Thermal Loop (HTL) in real time or Electrostatic QCD (EQCD) and Magnetostatic QCD (MQCD) in euclidean time and those describing processes in the medium like for example Soft Collinear Effective Field Theory (SCET) for jets and nonrelativistic EFTs like Non Relativistic QCD (NRQCD) or potential Nonrelativistic QCD (pNRQCD) for heavy quarks and quarkonium. In particular, EFTs allow to resum infinite class of diagrams related to the existence of a physical scale, to give a precise quantum field theoretical definition to objects of great phenomenological interest like the potential or the transport coefficients, to factorize contributions of different scales, to give a systematical framework for calculation of observables inside field theory. In this talk I will focus on heavy probes and examples of what discussed above will include the free energies, the heavy quark potential in medium, the heavy quark transport coefficients and the nonequilibrium evolution of quarkonium in the fireball.

To boost the EFT and lattice interface we founded the TUMQCD lattice collaboration [1-4]. In this talk I will review results at the interface of EFT and lattice.

\section{Heavy probes: Heavy Quarks and Quarkonium}

Heavy quarks are produced at the beginning of the collision and remain up to the end. The heavy-quark mass $m$ introduces a large scale, whose contribution may be factorized and computed in perturbation theory $\left(m \gg \Lambda_{Q C D}, \alpha_{s}(m) \ll 1\right)$. Low-energy scales are sensitive to the temperature $T$ and even if nonperturbative they may be accessible via lattice calculations. Quarkonia are special hard probes because being nonrelativistic bound states, they are multi-scale systems. They are endowed with three energy scales, the scale of the quark mass $m$ (hard scale), the scale of the momentum transfer $p \sim m v$ (soft) and the scale of the binding energy $E \sim m v^{2}$, being $v$ the velocity in the bound state and being $m v$ proportional to the reverse of the size $r$ of the system. If $v$ is smaller than 1 these scales are separated. In [5] it was summarized how to construct an effective field theory called potential NonRelativistic QCD (pNRQCD) that allows to define the potentials both in the case of a perturbative (weakly coupled pNRQCD) and of a nonperturbative soft scale (strongly coupled pNRQCD): they are the matching coefficients of the EFT and they are well defined in the 
matching procedure. pNRQCD has the Schrödinger equation as zero problem and it is constructed to be equivalent to QCD order by order in the expansion. In the case of strongly coupled pNRQCD the potentials are given in terms of generalized Wilson loops to be calculated on the lattice. In weakly coupled pNRQCD the degrees of freedom are color singlet and color octet quark-antiquark pair (together with US gluons), in strongly coupled pNRQCD we have only color singlets.

\section{1 pNRQCD at finite $T$}

Considering quarkonium in the hot QCD medium also the thermal scales of the Quark Gluon Plasma (QGP) are emerging: the temperature $T$, the Debye mass $m_{D} \sim g T$ related to the (chromo) electric screening and the scale $g^{2} T$ related to the (chromo)magnetic screening. In a weakly coupled plasma the scales are separated and hierarchically ordered. In a series of papers [6-11] a pNRQCD at finite $T$ description has been constructed.

\subsection{The Potential}

pNRQCD at finite $T$ allows us to give a clear and systematic definition of what is the quarkonium potential in medium. This has been investigated for years using many phenomenological assumptions, spanning from the internal energy to the free energy, either the average free energy or the singlet one which is gauge dependent. The EFT gives us for the first time the possibility to define what is this potential: it is the matching coefficient of the EFT that results from the integration of all the scales above the scale of the binding energy and it is the object that has to be inserted in the Schrödinger equation, the zero order equation in PNRQCD describing the real time evolution of the $Q \bar{Q}$ pair in medium. When $T$ is bigger than the energy, the potential depends on the temperature, otherwise not. Thermal effects appear in any case in the nonpotential contributions to the energy levels. We assumed that the bound state exist for $T \ll m$ and $1 / r \geq m_{D}$, we worked in the weak coupling limit and we consider all possible scales hierarchies [7]. We found that the thermal part of the potential has a real part (roughly described by the free energy) and an imaginary part. The imaginary part comes from two effects: the Landau damping [7, 11, 12], an effect existing also in QED, and the singlet to octet transition, existing only in QCD [7]. Which one dominates depends on the ratio between $m_{D}$ and $E$. In the EFT one could show that the imaginary part of the potential related to the Landau damping comes from inelastic parton scattering [9] and the singlet to octet transitition from gluon dissociation [10]. The existence of the imaginary part changed our paradigm for quarkonium suppression as the state dissociates for this reason well before that the conventional screening becomes active [7, 11, 12]. These large $T$ dependent imaginary parts call for an appropriate framework to describe the nonequilibrium evolution of quarkonium in medium: the open quantum system framework as we will discuss in Sec.4.

The pattern of thermal corrections is pretty interesting [7]: when $T<E$ thermal corrections are only in the energy; for $T>1 / r, 1 / r>m_{D}$ or $1 / r>T>E$ there is no exponential screening and $T$ dependent power like corrections appear; if $T>1 / r, 1 / r \sim m_{D}$ we have exponential screening but the imaginary part of the static potential is already bigger than the real one and dissociation already happened. Once the potential has been calculated, the EFT gives the systematic framework to obtain the thermal energies: in [6] it was performed the first QCD calculation of the thermal contributions to the $\Upsilon(1 S)$ mass and width at order $m \alpha_{s}^{5}$ at LHC below the dissociation temperature of about $500 \mathrm{MeV}$. This calculation is very important because it gives the parametric $T$ dependence 
of this observables. The width goes linear in $T$ in the dominant term and this has been confirmed by lattice calculations of the spectrum [18]. These findings in the EFT in perturbation theory have inspired many subsequent nonperturbative calculations of the static potential at finite $T$. In particular the Wilson loop at finite $T$ has been calculated on the lattice [23, 24] finding hints of a large imaginary parts. These calculations are pretty challenging and refining of the extraction methods are currently in elaboration [25, 27].

\section{Free Energies and Polyakov Loops}

Free energies and Polyakov loop calculations have been always very prominent in QCD at finite $T$, see e.g. the reviews [26, 29] Here, I report some recent developments at the interface of EFTs and lattice. We calculated the Polyakov loop and the Polyakov loop correlators both in perturbation theory and using EFTs to resum scales contributions in [30-33] and on the lattice to obtain these quantities fully nonperturbatively in [2,3]. In particular: the Polyakov loop has been computed up to order $g^{6}$, the (subtracted) $Q \bar{Q}$ free energy has been computed at short distances up to corrections of order $g^{7}(r T)^{4}, g^{8}$, the (subtracted) $Q \bar{Q}$ free energy has been computed at screening distances up to corrections of order $g^{8}$; the singlet free energy has been computed at short distances up to corrections of order $g^{4}(r T)^{5}, g^{6}$; the singlet free energy has been computed at screening distances up to corrections of order $g^{5}[30,31,33]$.

From the lattice simulations and from comparison to the perturbative results we could obtain the following important outcomes [2,3]: lattice calculations are consistent with weak-coupling expectations in the regime of application of the weakly coupled resummed perturbation theory which confirms the predictive power of the EFT; the crossover temperature to the quark-gluon plasma is $153+6.5-5 \mathrm{MeV}$ as extracted from the entropy of the Polyakov loop; the screening sets in at $r T \sim 0.3-0.4$ (observable dependent), consistent with a screening length of about $1 / m_{D}$; asymptotic screening masses are about $2 m_{D}$ (observable dependent); the first determination of the color octet $Q \bar{Q}$ free energy has been obtained [3] further investigated with a different setup in [28].

In the EFT/pNRQCD framework the Polyakov loop correlator $P_{c}$ can be decomposed as

$$
P_{c}(r, T)=\frac{1}{N_{c}^{2}}\left[e^{-f_{s}\left(r, T, m_{D}\right) / T}+\left(N_{c}^{2}-1\right) e^{-f_{o}\left(r, T, m_{D}\right) / T}+O\left(\alpha_{s}^{3}(r T)^{4}\right)\right]
$$

with $f_{s}=Q \bar{Q}$-color singlet free energy, $f_{o}=Q \bar{Q}$-color octet free energy to be matched from the singlet and octet $\mathrm{pNRQCD}$ propagators

$$
\frac{\left\langle S(\mathbf{r}, \mathbf{0}, 1 / T) S^{\dagger}(\mathbf{r}, \mathbf{0}, 0)\right\rangle}{\mathcal{N}}=e^{-V_{S}(r) / T}\left(1+\delta_{S}\right) \equiv e^{-f_{s}\left(r, T, m_{D}\right) / T}
$$

and

$$
\frac{\left\langle O^{a}(\mathbf{r}, \mathbf{0}, 1 / T) O^{a \dagger}(\mathbf{r}, \mathbf{0}, 0)\right\rangle}{\mathcal{N}}=e^{-V_{o}(r) / T}\left[\left(N_{c}^{2}-1\right)\left\langle P_{A}\right\rangle+\delta_{o}\right] \equiv\left(N_{c}^{2}-1\right) e^{-f_{o}\left(r, T, m_{D}\right) / T}
$$

where $V_{s}$ and $V_{O}$ are the singlet and octet static potentials, $\delta_{s}$ and $\delta_{o}$ stand for thermal loop corrections to the singlet/octet propagators, $\mathcal{N}$ is a normalization and $\left\langle P_{A}\right\rangle$ is the average value of an adjoint Wilson line. 
We may identify two possible regimes: low temperatures, $T \ll V_{s}\left(\right.$ or $\left.r T \ll \alpha_{s}\right),: P_{c} \approx \frac{e^{-V_{s} / T}}{N_{c}^{2}}$; high temperatures, $T \gg V_{s}\left(\right.$ or $\left.r T \gg \alpha_{s}\right): P_{c}$ is a linear combination of $e^{-f_{s} / T}$ and $e^{-f_{o} / T}$. A strict perturbative expansion in $\alpha_{s}$ corresponds to this regime. These regimes have been validated with the lattice calculations [3].

The free energies turn out not to be the objects to be used as a potential in the Schrödinger equation even if the singlet free energy may provide a good approximation of the real part of the static potential.

Differently from the Polyakov loop and the Polyakov loop correlator, the cyclic Wilson loop is divergent after charge and field renormalization. This divergence is due to intersection points [32]. In [32] it has been shown that this produces that the cyclic Wilson loop mixes under renormalization with the correlator of two Polyakov loops.The resulting renormalization equation has been tested up to order $g^{6}$ and used to resum the leading logarithms associated with the intersection divergence. The cyclic Wilson loop free energy has been computed at short distances up to corrections of order $g 5+L L$ resummation and a renormalization prescription relevant for lattice evaluation has been given [32].

\section{The quarkonium nonequilibrium evolution in medium: EFTs, Open Quantum Systems (OQS) and lattice}

The large imaginary parts appearing in the static potential motivated us to introducing an appropriate framework to describe the real time nonequilibrium evolution of quarkonium in the QGP medium. In [14-16] we have developed an open quantum system (OQS) framework (for OQS see [35] for a review and the seminal paper [36]) rooted in $\mathrm{NNRQCD}$ at finite $T$ that is fully quantum, conserves the number of heavy quarks and consider both color singlet and color octet quarkonium degrees of freedom. This has also been reported by the talk of Miguel Escobedo at this conference [34].

We distinguish the environment (QGP) characterized by the scale $T$ and the system (quarkonium) characterized by the scale $E$. We identify the inverse of $E$ with the intrinsic time scale of the system: $\tau_{S} \sim 1 / E$ and the inverse of $\pi T$ with the correlation time of the environment: $\tau_{E} \sim 1 /(\pi T)$. If the medium is in thermal equilibrium, or locally in thermal equilibrium, we may understand $T$ as a temperature, otherwise is just a parameter. The medium can be strongly coupled. The evolution of the system is characterized by a relaxation time $\tau_{R}$ that is estimated by the inverse of the color singlet self-energy diagram in pNRQCD at finite $T$. We select quarkonia states with a small radius (Coulombic) for which $1 / r \gg \pi T, \Lambda_{Q C D}$ and we consider $\pi T \gg E$.

In this framework ,in [14], a set of master equations governing the time evolution of heavy quarkonium in a medium were derived. The equations follow from assuming the inverse Bohr radius of the quarkonium to be greater than the energy scale of the medium, and model the quarkonium as evolving in the vacuum up to a time $t=t_{0}$ at which point interactions with the medium begin. The equations express the time evolution of the density matrices of the heavy quarkantiquark color singlet, $\rho_{s}$, and octet states, $\rho_{o}$, in terms of the color singlet and octet Hamiltonians, $h_{s}=\mathbf{p}^{2} / M-C_{F} \alpha_{s} / r+\ldots$ and $h_{o}=\mathbf{p}^{2} / M+\alpha_{s} /\left(2 N_{c} r\right)+\ldots$, and interaction terms with the medium, which, at order $r^{2}$ in the multipole expansion, are encoded in the self-energy diagrams of the EFTs. These interactions account for the mass shift of the heavy $Q \bar{Q}$ pair induced by the medium, its decay 
width induced by the medium, the generation of $Q \bar{Q}$ color singlet states from $Q \bar{Q}$ color octet states interacting with the medium and the generation of $Q \bar{Q}$ color octet states from $Q \bar{Q}$ (color singlet or octet) states interacting with the medium. The leading order interaction between a heavy $Q \bar{Q}$ field and the medium is encoded in PNRQCD in a chromoelectric dipole interaction, which appears at order $r / m^{0}$ in the EFT Lagrangian. The approach gives us master equations, in general non Markovian, for the out of equilibrium evolution of the color singlet and color octet matrix densities. The system is in non-equilibrium because through interaction with the environment (quark gluon plasma) singlet and octet quark-antiquark states continuously transform in each other although the total number of heavy quarks is conserved.

Assuming that any energy scale in the medium is larger than the heavy $Q \bar{Q}$ binding energy $E$, in particular that $\tau_{R} \gg \tau_{E}$, we obtain a Markovian evolution while the chosen hierarchy of scales implies $\tau_{s} \gg \tau_{E}$ qualifying the regime as quantum Brownian motion. In this situation we can reduce the general master equation to a Linblad form:

$$
\frac{\mathrm{d} \rho}{\mathrm{d} t}=-i[H, \rho]+\sum_{n}\left(C_{n} \rho C_{n}^{\dagger}-\frac{1}{2}\left\{C_{n}^{\dagger} C_{n}, \rho\right\}\right),
$$

where $H$ is a Hermitian operator, and $C_{n}$ are known as collapse operators. These operators were computed in $[14,15]$. If we assume an isotropic medium and the quarkonium at rest with respect to the medium, in the large time limit the equation 1 assumes a particular simple form:

$$
\begin{aligned}
\rho & =\left(\begin{array}{cc}
\rho_{s} & 0 \\
0 & \rho_{o}
\end{array}\right) \\
H & =\left(\begin{array}{cc}
h_{s} & 0 \\
0 & h_{o}
\end{array}\right)+\frac{r^{2}}{2} \gamma(t)\left(\begin{array}{cc}
1 & 0 \\
0 & \frac{7}{16}
\end{array}\right), \\
C_{i}^{0} & =\sqrt{\frac{\kappa(t)}{8}} r^{i}\left(\begin{array}{cc}
0 & 1 \\
\sqrt{8} & 0
\end{array}\right), \quad C_{i}^{1}=\sqrt{\frac{5 \kappa(t)}{16}} r^{i}\left(\begin{array}{cc}
0 & 0 \\
0 & 1
\end{array}\right)
\end{aligned}
$$

Interestingly enough in this case the properties of the QGP are encoded in two transport coefficients: the heavy quark momentum diffusion coefficient, $\kappa$, and its dispersive counterpart $\gamma$ which are given by time integrals of appropriate gauge invariant correlators at finite $T$ given by the integral of gauge invariant correlators of chromoelectric fields:

$$
\begin{aligned}
& \kappa=\frac{g^{2}}{6 N_{c}} \int_{0}^{\infty} \mathrm{d} s\left\langle\left\{E^{a, i}(s, \mathbf{0}), E^{a, i}(0, \mathbf{0})\right\}\right\rangle, \\
& \gamma=-i \frac{g^{2}}{6 N_{c}} \int_{0}^{\infty} \mathrm{d} s\left\langle\left[E^{a, i}(s, \mathbf{0}), E^{a, i}(0, \mathbf{0})\right]\right\rangle .
\end{aligned}
$$

In the case of a nonperturbative QGP, these objects are nonperturbative and should be evaluated on the lattice. Once the Linblad equation is solved and evolved up to freeze out one could obtain observables like the $R_{A A}$ and the $v_{2}$ by projecting over the quarkonium states of interest and compare to the experimental data at $\mathrm{LHC}$, see $[14,15]$.

Notice that in this case the OQS/PNRQCD framework allows us to use input from a lattice QCD in equilibrium calculation to describe the nonequilibrium evolution of quarkonium in medium. I $n$ 
[37] using the static limit the evolution equations have been obtained for quarkonia of any radius. In [42] we are devoloping a new computational approach to solve the Linblad equations in a more efficient way that allows to couple the quarkonium system to the full hydrodynamical evolution.

The semiclassical limit of similar equations have been studied in [17] and the relevance of correlated versus noncorrelated noise in [43]. In [38, 39] using this same pNRQCD and OQS framework and a particular scales hierarchy, transport equations have been obtained for the study of quarkonium in medium, in particular a semiclassical Boltzmann equation has been obtained and in the case of the differential reaction rate, the information on the QGP is contained in a novel chromoelectric gluon correlator involving also staple-shaped Wilson lines, in a way similar to what happens at $T=0$ in the gluon parton distribution functions, the gluon transverse momentum dependent parton distribution functions and in the quarkonium production cross section factorization in PNRQCD [40].

\section{Transport Coefficients $\kappa$ and $\gamma$}

The heavy quark momentum diffusion coefficient, $\kappa$, is an object of special interest in the literature, but one which has proven notoriously difficult to estimate, despite the fact that it has been computed by weak-coupling methods at next-to-leading order accuracy, and by lattice simulations of the pure SU(3) gauge theory. Another coefficient, $\gamma$, has been recently identified in the OQS description of quarkonium nonequilibrium evolution. It can be understood as the dispersive counterpart of $\kappa$. Nothing is known about $\gamma$, apart from its leading order, weak-coupling expression [7, 45]. Both $\kappa$ and $\gamma$ are, however, of foremost importance in heavy quarkonium physics as they entirely determine the in and out of equilibrium dynamics of quarkonium in a medium, if the evolution of the density matrix is Markovian, and the motion, quantum Brownian. The EFT allows to relate such coefficients to quarkonium thermal energy shifts and widths. Precisely [16], using quarkonia with a small radius (Coulombic) to probe the strongly coupled QGP, we get the relations

$$
\begin{gathered}
\Gamma=3<r>^{2} \kappa, \\
\delta M=\frac{3}{2}<r>^{2} \gamma,
\end{gathered}
$$

where $\Gamma$ and $\delta M$ are the thermal width and mass shift and $\langle r\rangle$ is the average radius of the given quarkonium state. Then, using $2+1$ flavors lattice calculations of $\Gamma$ and $\delta M[18,19]$ we could obtain the unquenched values for $\kappa$ and $\gamma$ shown in Figs. 1 and 2. In Fig.1, the first entry (black bar) shows $\kappa / T^{3}$ as obtained from Eq. (7) using the lattice data of Refs. [18, 19] for the lower and upper bounds of the thermal decay width of the $\Upsilon(1 S)$. The second entry (brown bar) reports the (quenched) lattice estimate of Ref. [20]. The third and fourth entries (green bars) are the determinations based on the ALICE [21] and STAR [22] measurements of the $D$-meson azimuthal anisotropy coefficient $v_{2}$, respectively. The fifth entry (blue bar) is the leading order (LO) perturbative result with the strong coupling computed at the scale $\pi \times(407 \mathrm{MeV})=1.28 \mathrm{GeV}$. We assign to it a $50 \%$ uncertainty.

In Fig. 2 the first three entries (black bars) show $\gamma / T^{3}$ as obtained from Eq. ((8)) using lattice data of Ref. [19] for the thermal mass shift of the $J / \psi$ and of the $\Upsilon(1 S)$ at two different temperatures. The error bars account for the lattice uncertainties only. The last two entries (blue bars) provide $\gamma / T^{3}$ in perturbation theory at leading order at two different temperatures. We assign 


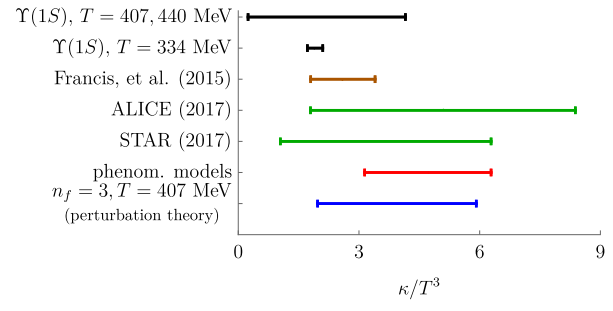

Figure 1: Extraction of $\kappa / T^{3}$, see the text for details.

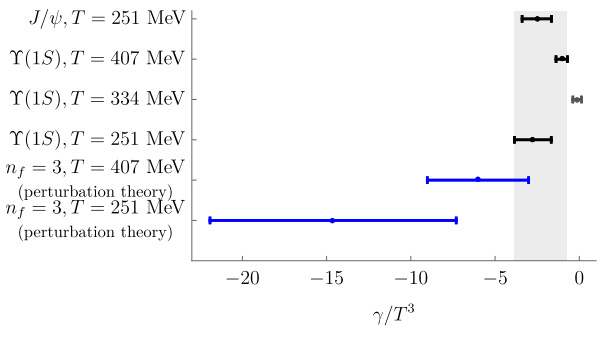

Figure 2: Extractions of $\gamma / T^{3}$, for details see the text.

a 50\% uncertainty to these results. The gray band gives our final range. The resulting range for $\kappa$ is consistent with the earlier determinations, the one for $\gamma$ is the first non-perturbative determination of this quantity.

This is a clear example of how one could obtain results beyond the present state of the art (in this case an unquenched lattice calculation of $\kappa$ and $\gamma$ ) taking advantage of the alliace between EFT and lattice.

\subsection{Direct lattice calculation of $\kappa$}

In [4] we computed the heavy quark momentum diffusion coefficient directly from the correlator of two chromo-electric fields attached to a Polyakov loop in pure SU(3) gauge theory.

Notice that in general the lattice calculations of the transport coefficients are very difficult. In fact, to obtain the transport coefficients one has to reconstruct the spectral functions from the appropriate Euclidean time correlation functions. At low energies, $\omega$, the spectral function has a peak, called the transport peak, and the width of the transport peak defines the transport coefficient. Thus, one needs a reliable determination of the width of the transport peak in order to obtain the transport coefficient from lattice QCD calculations, which is difficult. In the case of heavy quarks, this is even more challenging because the width of the transport peak is inversely proportional to the heavy quark mass. Moreover, Euclidean time correlators are rather insensitive to small widths. The above difficulty in the determination of the heavy quark diffusion coefficient can be circumvented by using EFTs. Namely, by integrating out the heavy quark fields one can relate the heavy quark diffusion coefficient to the correlator of the chromoelectric field strength, as we have previously discussed. The corresponding spectral function does not have a transport peak and the small $\omega$ behavior is smoothly connected to the UV behavior of the spectral function The heavy quark diffusion coefficient is given by the intercept of the spectral function at $\omega=0$ and no determination of the width of the transport peak is needed. . Recently the subleading correction (in the mass expansion) to $\kappa$ has been calculated and found to be proportional to a correlator of magnetic fields [44].

Using a multilevel algorithm and tree-level improvement, we studied the behavior of the diffusion coefficient as a function of temperature in an unprecedented wide range of temperature $1.1<T / T_{c}<10^{4}$. At high $\mathrm{T}$ is possible to compare with the perturbative expansions in the EFT and we find that within errors the lattice results are remarkably compatible with the next-to-leading order perturbative result, as you see from Fig. 3 . 
These results expose for the first time the temperature dependence of $\kappa$ in a large of window of temperature and have a great impact on $R_{A A}$ and $v_{2}$ predictions and it has been shown solving the Linblad equations of [14, 15] with a $T$ dependent $\kappa$, cf. [42].

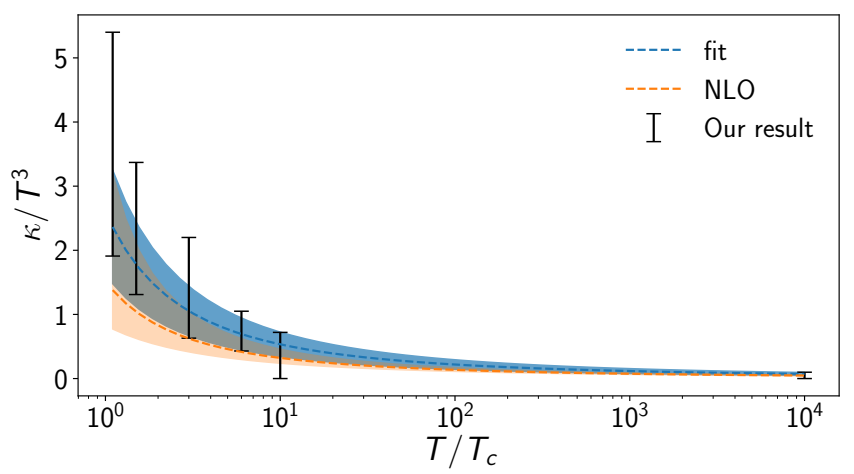

Figure 3: Temperature dependence of our results compared to the NLO result. The shaded bands include the errors coming from varying the scale by a factor 2 . The blue band also includes the statistical error.

This correlator has been recently computed quenched on the lattice at $T=1.5 T_{c}$ using the gradient flow in [41].

\section{Outlook}

We focused on heavy quarks but there are other hard probes to which the EFT/lattice approach can be applied. A clear example is the calculation of $\hat{q}$ that controls jet quenching. A systematic treatment of a complex phenomenon like jet quenching is possible in an EFT framework owing to the hierarchy of scales that characterizes the system. These are typically SCET scales $Q, Q \lambda, Q \lambda^{2}$ with $\lambda=T / Q$ which characterize the propagation of a very energetic parton in the medium and the thermal scales that characterize the medium itself. $\hat{q}$ is the jet quenching parameter, i.e. the mean square transverse momentum picked up by the hard parton per unit distance traveled. Using EFT methods $\hat{q}$ can be expressed in terms of gauge invariant gauge field correlators to be calculated on the lattice, see $[46,47]$. Recently an open quantum system description of jet quenching rooted in the EFT/lattice has been developed [48] in analogy to what I have described above for the nonequilibrium evolution of quarkonium in medium.

In summary, the alliance of EFTs, resummed perturbative and lattice methods allows to study hard probes directly in the realm of $Q C D$ in a systematic way. In this framework hard probes become a unique laboratory for the study of the QGP. Some previous disagreements between perturbation theory and lattice appear to be solved. The EFT factorization at lower energy increase our predictivity power: nonperturbative objects depends only on the lower energy scale, are reduced in number and formulated such that they can be evaluated on the lattice directly. EFTs allow us to give the appropriate definition and define a calculational scheme for quantities of huge phenomenological interest. EFTs allow us to enlarge the applicability region of lattice see e.g. the non equilibrium evolution of quarkonium in the fireball. I have reported results on the free energies, the potential, the thermal spectrum, the $R_{A A}$, some diffusion coefficient but more can be studied in the same framework. Many of the correlators factored in the EFT have still to be computed on the lattice. 
Calculating these objects on the lattice and developing tools to calculate them also unquenched, like the gradient flow, as well as efficient techniques to relate to continuum will have a profound impact on the phenomenology of hard probes. Many EFTs applications have still to be worked out and new field entered like nonequilibrium studies with open quantum systems.

\section{References}

[1] https://einrichtungen.ph.tum.de/T30f/tumqcd/index.html

[2] A. Bazavov, N. Brambilla, H. T. Ding, P. Petreczky, H. P. Schadler, A. Vairo and J. H. Weber, Phys. Rev. D 93 (2016) no.11, 114502 [arXiv:1603.06637 [hep-lat]].

[3] A. Bazavov et al. [TUMQCD], Phys. Rev. D 98 (2018) no.5, 054511 [arXiv:1804.10600 [hep-lat]].

[4] N. Brambilla, V. Leino, P. Petreczky and A. Vairo, [arXiv:2007.10078 [hep-lat]].

[5] N. Brambilla, A. Pineda, J. Soto and A. Vairo, Rev. Mod. Phys. 77 (2005), 1423 [arXiv:hep$\mathrm{ph} / 0410047$ [hep-ph]].

[6] N. Brambilla, M. A. Escobedo, J. Ghiglieri, J. Soto and A. Vairo, JHEP 09 (2010), 038 [arXiv:1007.4156 [hep-ph]].

[7] N. Brambilla, J. Ghiglieri, A. Vairo and P. Petreczky, Phys. Rev. D 78 (2008), 014017 [arXiv:0804.0993 [hep-ph]].

[8] S. Biondini, N. Brambilla, M. A. Escobedo and A. Vairo, Phys. Rev. D 95 (2017) no.7, 074016 [arXiv:1701.06956 [hep-ph]].

[9] N. Brambilla, M. A. Escobedo, J. Ghiglieri and A. Vairo, JHEP 05 (2013), 130 [arXiv:1303.6097 [hep-ph]].

[10] N. Brambilla, M. A. Escobedo, J. Ghiglieri and A. Vairo, JHEP 12 (2011), 116 [arXiv:1109.5826 [hep-ph]].

[11] M. A. Escobedo and J. Soto, Phys. Rev. A 78 (2008), 032520 doi:10.1103/PhysRevA.78.032520 [arXiv:0804.0691 [hep-ph]]; M. A. Escobedo and J. Soto, Phys. Rev. A 82 (2010), 042506 [arXiv:1008.0254 [hep-ph]].

[12] M. Laine, O. Philipsen, P. Romatschke and M. Tassler, JHEP 03 (2007), 054 [arXiv:hepph/0611300 [hep-ph]].

[13] P. Petreczky, A. Rothkopf and J. Weber, Nucl. Phys. A 982 (2019), 735-738 [arXiv:1810.02230 [hep-lat]].

[14] N. Brambilla, M. A. Escobedo, J. Soto and A. Vairo, Phys. Rev. D 97 (2018) no.7, 074009 [arXiv:1711.04515 [hep-ph]]. 
[15] N. Brambilla, M. A. Escobedo, J. Soto and A. Vairo, Phys. Rev. D 96 (2017) no.3, 034021 [arXiv: 1612.07248 [hep-ph]].

[16] N. Brambilla, M. A. Escobedo, A. Vairo and P. Vander Griend, Phys. Rev. D 100 (2019) no.5, 054025 [arXiv: 1903.08063 [hep-ph]].

[17] J. P. Blaizot and M. A. Escobedo, JHEP 06 (2018), 034 [arXiv:1711.10812 [hep-ph]]; J. P. Blaizot and M. A. Escobedo, Phys. Rev. D 98 (2018) no.7, 074007 [arXiv:1803.07996 [hep-ph]].

[18] G. Aarts, C. Allton, S. Kim, M. P. Lombardo, M. B. Oktay, S. M. Ryan, D. K. Sinclair and J. I. Skullerud, JHEP 11 (2011), 103 [arXiv:1109.4496 [hep-lat]].

[19] S. Kim, P. Petreczky and A. Rothkopf, JHEP 11 (2018), 088 [arXiv:1808.08781 [hep-lat]].

[20] A. Francis, O. Kaczmarek, M. Laine, T. Neuhaus and H. Ohno, Phys. Rev. D 92 (2015) no.11, 116003 [arXiv:1508.04543 [hep-lat]].

[21] S. Acharya et al. [ALICE], Phys. Rev. Lett. 120 (2018) no.10, 102301 [arXiv:1707.01005 [nucl-ex]].

[22] L. Adamczyk et al. [STAR], Phys. Rev. Lett. 118 (2017) no.21, 212301 doi:10.1103/PhysRevLett.118.212301 [arXiv:1701.06060 [nucl-ex]].

[23] A. Rothkopf, T. Hatsuda and S. Sasaki, Phys. Rev. Lett. 108 (2012), 162001 [arXiv:1108.1579 [hep-lat]].

[24] A. Rothkopf, Phys. Rept. 858 (2020), 1-117 [arXiv:1912.02253 [hep-ph]].

[25] P. Petreczky, A. Rothkopf and J. Weber, Nucl. Phys. A 982 (2019), 735-738 [arXiv:1810.02230 [hep-lat]].

[26] J. Ghiglieri, A. Kurkela, M. Strickland and A. Vuorinen, Phys. Rept. 880 (2020), 1-73 [arXiv:2002.10188 [hep-ph]].

[27] D. Bala and S. Datta, Phys. Rev. D 101 (2020) no.3, 034507 [arXiv:1909.10548 [hep-lat]].

[28] D. Bala and S. Datta, [arXiv:2009.00773 [hep-lat]].

[29] A. Bazavov and J. H. Weber, [arXiv:2010.01873 [hep-lat]].

[30] M. Berwein, N. Brambilla, P. Petreczky and A. Vairo, Phys. Rev. D 96 (2017) no.1, 014025 [arXiv:1704.07266 [hep-ph]].

[31] M. Berwein, N. Brambilla, P. Petreczky and A. Vairo, Phys. Rev. D 93 (2016) no.3, 034010 [arXiv:1512.08443 [hep-ph]].

[32] M. Berwein, N. Brambilla and A. Vairo, Phys. Part. Nucl. 45 (2014) no.4, 656-663 [arXiv:1312.6651 [hep-th]]. 
[33] N. Brambilla, J. Ghiglieri, P. Petreczky and A. Vairo, Phys. Rev. D 82 (2010), 074019 [arXiv: 1007.5172 [hep-ph]].

[34] Miguel Escobedo, Talk "Transport coefficients from in medium quarkonium dynamics", presented at Hard Probes.

[35] Y. Akamatsu, [arXiv:2009.10559 [nucl-th]].

[36] Y. Akamatsu, Phys. Rev. D 91 (2015) no.5, 056002 [arXiv:1403.5783 [hep-ph]].

[37] M. Á. Escobedo, [arXiv:2010.10424 [hep-ph]].

[38] X. Yao, W. Ke, Y. Xu, S. A. Bass, T. Mehen and B. Müller, [arXiv:2002.04079 [hep-ph]]; X. Yao and T. Mehen, [arXiv:2009.02408 [hep-ph]].

[39] X. Yao and T. Mehen, Phys. Rev. D 99 (2019) no.9, 096028 [arXiv:1811.07027 [hep-ph]].

[40] N. Brambilla, H. S. Chung and A. Vairo, [arXiv:2007.07613 [hep-ph]].

[41] L. Altenkort, A. M. Eller, O. Kaczmarek, L. Mazur, G. D. Moore and H. T. Shu, [arXiv:2009.13553 [hep-lat]].

[42] N. Brambilla, M. Escobedo, M. Strickland, P. Vander Griend, A. Vairo, J. Weber, in preparation.

[43] R. Sharma and A. Tiwari, Phys. Rev. D 101 (2020) no.7, 074004 [arXiv:1912.07036 [hep-ph]].

[44] A. Bouttefeux and M. Laine, [arXiv:2010.07316 [hep-ph]].

[45] A. M. Eller, J. Ghiglieri and G. D. Moore, Phys. Rev. D 99 (2019) no.9, 094042 [erratum: Phys. Rev. D 102 (2020) no.3, 039901] [arXiv:1903.08064 [hep-ph]].

[46] M. Benzke, N. Brambilla, M. A. Escobedo and A. Vairo, JHEP 02 (2013), 129 [arXiv:1208.4253 [hep-ph]]; M. Laine, Eur. Phys. J. C 72 (2012), 2233 [arXiv:1208.5707 [hep-ph]]; A. Majumder, Phys. Rev. C 87 (2013), 034905 [arXiv:1202.5295 [nucl-th]]; M. Panero, K. Rummukainen and A. Schäfer, Phys. Rev. Lett. 112 (2014) no.16, 162001 [arXiv: 1307.5850 [hep-ph]].

[47] A. Kumar, A. Majumder and J. H. Weber, [arXiv:2009.05655 [nucl-th]].

[48] V. Vaidya and X. Yao, [arXiv:2004.11403 [hep-ph]] 